obvious if the child is old enough to stand because the lordotic stance accentuates the disability. Aerophagy and simple constipation are other causes of gaseous distension of the abdomen; while chronic intestinal obstruction by tuberculous peritonitis or chronic intussusception is occasionally responsible.

When there is an acute gaseous distension of the abdomen the cause may be a relatively mild one, e.g. acute indigestion, but is more probably dangerous; for instance, acute intestinal obstruction or acute peritonitis. Some reference has been made to these conditions in an earlier section; here it will be sufficient to add that congenital atresia of the bowel and peritonitis secondary to umbilical sepsis are causes which may be found in the neonatal period. It is noteworthy that in some cases of acute pneumonia and pericarditis the alimentary tract becomes greatly distended with gas, and differential diagnosis from an acute abdominal lesion may be difficult at first.

Faecal accumulation is seldom great enough to cause obvious abdominal swelling, but sometimes even a simple constipation will do so. More often one finds megacolon or coeliac disease. In mentioning the latter it must not be assumed that the accumulation of large quantities of soft fatty faeces in the bowel is the sole cause of the abdominal swelling, but it certainly plays a part. The diagnosis is seldom difficult if the child's history is carefully taken, and if one notes the wasting of trunk and limbs, flatness of buttocks and distinctive character of the motions.

Distension of the abdomen by peritoneal effusion is relatively uncommon in early childhood, tuberculous peritonitis being undoubtedly the most frequent cause; nephritis, cardiac failure, severe anaemias, and hepatic cirrhosis are occasionally responsible. In some infants with simple indigestion and atony of intestinal and abdominal muscles there may be a sufficient accumulation of fluid within the alimentary tract to simulate shifting dulness, and so the diagnosis of ascites may be made incorrectly.

Finally, swelling of the abdomen may be caused by hepatomegaly, splenomegaly, an ovarian cyst, a dermoid, or a malignant tumour, e.g. renal, adrenal, or retroperitoneal.

\title{
REFERENCES
}

(x) CAPON, N. B., "The Examination of Sick Children," being Chap. XI in Symptoms and Signs in Clinical Medicine by E. Noble Chamberiain." Bristol, r938.

(2) CAPON, N. B., "Malnutrition in Children," Med. Press and Circular, 194I, ccv. 4I7.

(3) MARRIOTT, W̆. McK., and P. C. JEANS, Infant Nutrition, London, I942.

\section{INTRA-THORACIC TUBERCULOSIS IN CHILDHOOD}

\author{
By WILFRID SHELDON, M.D., F.R.C.P. \\ Physician to Children's Dept., King's College Hospital; Physician to Out-Patients, Hospital for \\ Sick Children, Great Ormond Street.
}

It is common knowledge that since the war there has been a considerable increase in the incidence of tuberculosis. This has been caused in part by a rise in milk-borne infection, but mainly by an increase in pulmonary disease. Children have borne an unexpectedly heavy share in this increase and the death-rate from tuberculous meningitis in children thas risen by as much as 50 per cent. Recently the Chief Medical Officer to the London Countv Council has reported (1) that the death-rate from pulmonary tuberculosis in I94I as compared with 1938 has increased by 442 per cent in children between birth and four years of age, and by 340 per cent in children between five and fourteen years of age. In view of this very considerable increase I have thought it fitting in this article to review our knowledge of intra-thoracic tuberculosis in childhood.

\section{Mode of Infection.}

Children acquire intra-thoracic tuberculosis as the result of inhaling the infecting grganism from an adult who is coughing a positive sputum. It is rare for one child to infect another child, because the pathological process in a child differs from that in an adult, and the pro- 
duction of sputum is an infrequent symptom; and further, those children who produce sputum habitually swallow it rather than expectorating. Occasionally as puberty approaches the course of events in a child's lung may conform to that met with in adults, with the formation of a cavity and the production of a good deal of positive sputum, and such a child can, of course, infect others, but these cases are so few as hardly to affect the statement that children are almost entirely infected by adults. It must be remembered that young children are probably more easily infected than adults because they have had no opportunity of developing any previous immunity, and for the same reason once infection has occurred in them it is more liable to generalise, giving rise to such fatal types of infection as miliary tuberculosis and tuberculous meningitis. So easy is it for a child to become infected when exposed to a phthisical adult that when such a history is obtained it is wise to assume that the child has been infected until the contrary can be proved.

At the present time the public are but little aware of the real danger of allowing children to be exposed to adults with pulmonary tuberculosis, and our profession has been slow to put into practice the methodical search for and examination of young children who have been exposed to adults known to be infected, but the prevention of intra-thoracic tuberculosis in children, or at any rate its early detection, is not likely to become effective until these measures are more widely and more energetically adopted. Another important point with regard to infection which is apt to be overlooked is that the length of time for which a child needs to be exposed to an adult in order to become infected may be very short, as short as one day. The importance of realising this lies in the fact that for a few weeks preceding the diagnosis and notification of disease in an adult, children other than those in the immediate family may have been in contact with the adult patient, perhaps only for a day or two, and yet may have become infected in that time, and an efficient follow-up of child contacts must inevitably range beyond the immediate members of the family.

The question of child contacts has been recently investigated by my colleague, Dr. Marcia Hall, to whom I am indebted for some of the following case histories. A careful perusal of them will reveal the tragedy of allowing children to be exposed to adults with phthisis.

$J$. G., aged three months on admission to hospital with miliary tuberculosis. Died the following month. A sister, $N$. G., aged four years, was admitted to hospital a month later with tuberculous mediastinal glands and made a slow recovery. There was a history that both children had spent one night in a house with their phthisical aunt. No other history of contact could be obtained.

$S$. $F$., aged two years, admitted to hospital with miliary tuberculosis and died the following month. This child had spent one afternoon nine months previously in close contact with her father before his admission to a sanatorium. She had not been in contact with him previously, and no other history of contact could be obtained.

These two histories illustrate the brevity of exposure which is necessary in order to infect a child.

Six healthy children were evacuated to the country and billeted with a woman known to have been coughing a positive sputum some years before. During their stay in this foster-home five of the children developed intra-thoracic tuberculosis. Their infection was brought to light because one of them was admitted to hospital with phlyctenular conjunctivitis, and this led to the examination of the other children.

$R$. $P$., aged four years, and $J . P$., aged six years, were living with their mother who was known to have a tuberculous cavity at the right apex, and who attended a tuberculosis dispensary. Both children had tuberculous infection of their mediastinal glands.

Three young boys in a family spent the nights of the winter of I940-4I in a private air-raid shelter which they shared with their neighbours. Of the neighbours, the middleaged father had phthisis and had infected his grown-up son and daughter, their infection being discovered as a result of routine examination of contacts. The three brothers, not being in the infected family, were not examined until the mother brought one to hospital for a different reason. When the history of contact had been ascertained the three young boys were examined and all were in the process of recovery from intra-thoracic tuberculosis.

This last case history demonstrates the necessity for looking beyond the immediate family when child contacts of a known phthisical adult are being searched for. 


\section{Pathology.}

That tuberculous infection in the chest in childhood takes a different pathological course as compared with an adult has already been mentioned. Actually in a child two separate processes are at work following infection with the tubercle bacillus. One concerns the slow advance of the invading organism and to check this the child laboriously accumulates an immunity mechanism. The other process, which has its own clinical manifestations, consists of the development of a state of sensitivity to the tuberculo-protein (tuberculin) liberated from the bodies of destroyed organisms. These two processes must be described separately, at the same time recounting the symptoms and signs which may occur, but the reader must appreciate that in actual fact both processes are running simultaneously. These two sets of events will be described under the headings "Stages of Infection" and "Development of Sensitivity."

Stages of Infection.-The tubercle bacillus, on being inhaled into the lungs, is drawn into a terminal bronchiole usually situated near the surface of the lung and penetrates the bronchial mucosa. There it acts as a foreign substance to which the body responds with a cellular reaction-giant cells, plasma cells, lymphocytes-forming the well-known histological features of a tubercle. The importance of this initial lesion was first appreciated by Ghon, and is spoken of as the "primary focus of Ghon." It may give rise to no symptoms or signs but tends slowly to heal by a combined process of fibrosis and calcification. If symptoms arise they are not likely to amount to more than a dry cough persisting for some weeks, a low temperature of one or two degrees above normal, and a temporary failure to gain weight. The lesion may be no larger than a split pea, and is too small to detect by the ordinary clinical means.

If the disease spreads from the primary focus the course of events at once differs from that seen in an adult, in whom the disease tends to spread locally and centrifugally, going on to caseation and maybe cavity formation. This is seldom seen in a child, in whom the primary focus does not tend to spread locally, but the infection passes along the lymphatics which lie alongside the bronchial tree. Thus the organisms pass from one peribronchial gland to the next, until they reach the group of glands at the roots of the lungs-the mediastinal glands. At this point the disease has reached its second stage, and involvement of the mediastinal glands together with the primary focus is sometimes spoken of as the "primary complex."

As in the case of a primary focus, so infection of the mediastinal glands may pass unnoticed, and a chance X-ray taken some years later may show old calcified areas in the mediastinal glands. Should symptoms arise, they have no outstanding or characteristic features. A dry cough may arise, and may give more trouble at night than by day. The temperature may be found raised to $100^{\circ} \mathrm{F}$. or so, and the child's weight cease to increase. During the first year or two of life, when the thoracic cavity is small, enlarging mediastinal glands may press on the trachea or main bronchi and give rise to a stridor which may become severe, tends to be continuous, and will last for several months until healing of the glands takes place. It follows that stridor appearing some months after birth should arouse a suspicion of tuberculous mediastinal adenitis.

The physical signs of enlarged mediastinal glands are usually vague and difficult to elicit, indeed physical signs may be absent. One of the most characteristic signs, described by d'Espine, is obtained by auscultating the whispered voice sound down the thoracic spine. In health pectoriloquy from the trachea is heard down to the second thoracic spine, but if the gland in the bifurcation of the trachea be enlarged the tracheal pectoriloquy may be heard as low as the fourth, fifth or sixth spine. It will be appreciated that a positive d'Espine's sign is not diagnostic of tuberculosis, but simply of an enlarged bifurcation gland. Impairment of the percussion note between the scapulae or on either side of the manubrium sterni, although described, can seldom be demonstrated. Should the main bronchi be compressed, air entry into the lung beyond may become defective leading to a flattening of the percussion note and diminished breath sounds, and such areas are most likely to be discovered in one or other axillae, or close to the spine from the root of the lung down to the diaphragm. In young children an area of lung may become collapsed by this mechanism, and the mediastinum be displaced towards the collapsed area. On the other hand, severe pressure on a bronchus may lead to an exactly opposite state of affairs in which the bronchus opens to admit air, but egress of air is prevented, giving rise to a condition of obstructive emphysema with a hyper-resonant percussion note, diminished breath sounds, and displacement of the mediastinum away from the affected side. Occasionally pressure at the root of the lung may cause a local catarrh in the bronchi giving moist sounds over the roots of the lung-a condition called "hilum catarrh." 
The majority of children with infection of the mediastinal glands make a slow recovery, the organisms becoming hemmed in by fibrosis and incarcerated by calcification. For the disease to go on to a fatal outcome a further extension of the disease is necessary. This may occur as a result of a mediastinal gland softening and eventually leaking into a bronchus, the contents of the gland being inhaled and setting up a tuberculous broncho-pneumonia. Rarely one of these glands may burst into the oesophagus or aorta. An alternative method of extension is by a gradual spread of infection ascending the para-tracheal glands until the organisms pass beyond the top gland into the right lymphatic trunk or the thoracic duct and thus gaining the blood stream. The events that follow this will depend upon the number of organisms reaching the blood stream. If the number is small infection may appear in some distant part, such, for example, as in a joint or kidney; if the number is great, generalised miliary tuberculosis will develop, with fatal results.

Development of Sensitivity.-It is generally considered that a time interval of about six weeks to three months occurs between the primary infection and the involvement of the mediastinal glands, and it so happens that the same period sees the development of sensitivity to tuberculin. Such sensitivity may produce local effects in the lungs or local effects in distant parts, but is invariably accompanied by the capacity to give a positive skin reaction to tuberculin tests. The local results of sensitivity in the chest are for the most part demonstrable by X-rays, and appear as a "hilum flare," which is shown as a triangular shadow with its apex at the root of the lung. Although the shadow may suggest that a considerable area of lung is diseased, the shadow slowly retrogresses over a period of months. Occasionally a benign consolidation of as much as a whole lobe of a lung develops as a local manifestation of sensitivity and to this the name of epituberculosis is given. In such a case the child may present physical signs such as are obtained in lobar pneumonia. The outlook in these children is ultimately good, but it may be a matter of months before resolution is complete. Some authorities consider that the development of pleurisy with effusion is also a manifestation of sensitivity; whether this be so or not, it is true that tuberculous pleurisy with effusion in children generally arises shortly after the initial infection, and by making for an early diagnosis and by getting the child under early treatment the outlook is generally favourable.

Distant manifestations of sensitivity only arise in probably a small proportion of infected children, but they are of great importance as they should warn the doctor that a recent tuberculous infection has taken place. These manifestations are two in number-erythema nodosum and phlyctenular conjunctivitis.

Erythema nodosum consists of the appearance of red, raised, slightly tender nodes up to half an inch or more in diameter, down the shins and occasionally above the knee and on the forearm. While they are present the temperature is raised three or four degrees. With rest in bed they gradually subside after two or three weeks, leaving some staining of the skin. These nodes do not suppurate. It must be understood that erythema nodosum is not due to the presence of tubercle bacilli in these nodes, but is actually an expression of the recent development of bacterial sensitivity; and the tubercle bacillus is not the only organism that can cause the condition, streptococci, meningococci, and other organisms being also capable of reproducing the disease. Tuberculosis is, however, the main cause and accounts for roughly three-quarters of the cases. Merely to diagnose erythema nodosum, to put the child to bed and to give a reassuring prognosis is not sufficient in the light of our knowledge. It is essential to enquire further, and by every available means, whether the child has been recently infected by tuberculosis.

Phlyctenular conjunctivitis in its significance is comparable with erythema nodosum, again about three-quarters of the cases being associated with recent tuberculosis. The phlyctenule appears as a little raised yellowish nodule of pinhead size on the conjunctiva close to the corneo-scleral junction, and a leash of minute vessels can usually be made out running up towards the phlyctenule. It gives rise to local irritation, persists for some few weeks, and fresh phlyctenules may appear over a period of months.

In the case histories given earlier it may be recalled that infection of five children evacuated in one billet was discovered because one of them attended hospital with phlyctenular conjunctivitis. A case history which illustrates the importance of erythema nodosum is the following: a child, aged four years, was taken to a boarding house at Brighton for a holiday. $\mathrm{He}$ played on the beach with a young man staying at the boarding-house, who, after a few days had a sudden haemoptysis and was subsequently removed to a sanatorium. Two months 
later the child developed erythema nodosum, the significance of which was missed. After a further three months the child was brought to hospital with tuberculous broncho-pneumonia which proved fatal.

\section{Diagnosis.}

It must be admitted that the diagnosis of intra-thoracic tuberculosis in children, if reliance is to be placed solely on symptoms and signs, is a matter of extreme difficulty and often impossibility-unless one is prepared to wait until the disease is approaching a hopeless stage. Equally to wait for confirmation of the diagnosis until the child produces a positive sputum would be a policy fraught with disaster. It is no reflection on clinical medicine to state that for the early diagnosis of this disorder ancillary investigations, particularly skin tests and X-rays, are essential.

Skin tests.-Either the Mantoux intradermal or the Vollmer patch tests are employed. The former consists of the intradermal injection of $0 \cdot I$ c.c. of $I /$ Iooo old tuberculin, a positive result being shown by the development of a raised, red, indurated area half an inch or more in diameter after an interval of forty-eight hours. The patch test is performed by cleaning the skin with ether and applying a strip of sticky plaster on which are three squares of filter paper, the two outer squares containing tuberculin and the centre square acting as a control. The patch is removed after forty-eight hours, and twenty-four hours later the skin is examined, when a positive test is shown by a raised, red area opposite either or both of the tuberculin squares. The test fades in about a week. In the author's experience the Mantoux test is more delicate and gives a slightly higher percentage of positive reactions, but has the disadvantage that it may antagonise a nervous child, and occasionally the local reaction may be unpleasantly severe. As to the interpretation of these tests, a positive finding under three years of age almost invariably means the presence of an active tuberculous lesion somewhere in the body. After three years of age an increasing percentage of positive tests are the result of a previous and healed infection, although there is some evidence that the more severe the reaction the more likely is the tuberculous lesion to be still active. Technical errors account for a small number of negative findings; and children who are severely ill, whether from advanced tuberculosis or other disease, are likely to give negative reactions. It will be apparent that skin tests find their greatest value in young children; and certainly the patch test, which is easy of application, should be employed much more frequently than is the case at the present day.

$X$-rays.-Satisfactory X-rays of young children call for considerable technical skill, and the apparatus must be one which will take a good picture on a short exposure, because young children will seldom hold their breath. From the nature of the pathological changes the principal findings are usually at one or both hila, an area where interpretation of shadows is difficult and calls for much experience. A true lateral view of the chest is often helpful, for in that position enlargement of glands round the bifurcation of the trachea may be identified when in an antero-posterior view they are concealed behind the heart shadow. Often the position of enlarged glands and the direction of their advance out from the hilum will indicate the area of lung in which the primary focus may be expected. The primary focus is sometimes surrounded by a zone of sensitised and consolidated lung, which may give rise to a shadow on the film; otherwise the primary focus is not likely to be seen unless it has actually commenced to calcify.

To summarise, a child who is known to have been in contact with a phthisical adult must always be suspected of having acquired tuberculous infection, and a negative clinical examination is not sufficient to exclude a positive diagnosis. Investigation by means of X-rays must be carried out, and in young children as great, if not greater, importance, attaches to the result of skin tests, and both these investigations must be negative before assuming that a child exposed to the possibility of infection has in fact escaped.

\section{Prognosis.}

The outlook depends principally on the stage of the disease when the diagnosis is reached, but age is an important factor as well, for the younger the child the more rapid is the course of the illness and the greater is the risk of the infection becoming generalised. This is borne out by the fact that death from tuberculous meningitis reaches its highest incidence in the second year of life. In practice it is seldom that the diagnosis is made before the mediastinal 
glands are involved, and if X-rays indicate that the infection is already passing up the paratracheal glands the outlook must be guarded. In a young child the infection has also reached an ominous stage if stridor is heard. Apart from these considerations the majority of children with infected mediastinal glands make a gradual recovery. A cessation of fever is favourable, but even better prognostic import should be attached to a steady gain in weight. In gauging the course of the illness much help is given by repeated estimations of the sedimentation rate of the red cells, which may with advantage be repeated at fortnightly or monthly intervals. It must be remembered that the sedimentation rate is affected by any infective process, and it is therefore essential to exclude conditions such as naso-pharyngeal sepsis before assuming that the sedimentation rate is being entirely influenced by the tuberculous process. The sedimentation rate will also offer a guide as to when the child may be allowed up and about, for complete rest should be maintained until the rate has fallen to $12 \mathrm{~mm}$. or less in one hour.

\section{Treatment.}

This consists of complete rest in bed so long as the sedimentation rate is raised, but there is no need to confine the child to one room nor to being indoors, for open-air rest has a value of its own. The diet should be as nourishing as possible, and indigestible articles must be avoided, but there is no need for various extras bearing some mystical and fictitious nutritional value to be lavished on the child. Calcification plays such an important part in the healing of the lesions in children that an extra supply of calcium may be given such as 15 gr. calcium gluconate or a teaspoonful of syrup of calcium lacto-phosphate thrice daily. To ensure the utilisation of the calcium, vitamin $\mathrm{D}$ should be given, and may be combined with vitamin $\mathrm{A}$ in the form of halibut liver oil, Io drops daily, or an equal amount of one of the concentrates of these vitamins.

Lastly, the general health of the child must be as good as possible, and such common adverse influences as chronic naso-pharyngeal sepsis, dental infections, indigestion, and constipation must be properly dealt with.

\section{REFERENCE}

(I) DALEY, W. A., and BENJAMIN, B., B.M.J., 42. 2.417.

\section{NEONATAL MORTALITY}

\section{A report of the Sub-committee of the British Paediatric Association}

I. We wish to make it clear, at the outset, that when we speak of neonatal mortality we have also in mind $(a)$ the no less important, though less sharply defined, question of neonatal morbidity; and $(b)$ the loss of potential lives by stillbirth. In regard to neonatal morbidity, it should be stressed that though young infants possess a remarkable power of recovery from trauma and illness, failure to make full recovery is especially regrettable in early life, when each individual should be entitled to the prospect of a long term of good health.

2. It is common knowledge that the gratifying decline in the infant mortality rate which has taken place in most countries during the past fifty years or more, has not been accompanied by a correspondingly satisfactory fall of the neonatal mortality rate. Yet some countries and areas (e.g. Holland, Australasia, and the large cities of the U.S.A.) have been more successful in this respect than others (e.g. Scotland, England and Wales), which shows that the problem is not insoluble.

3. In studying neonatal mortality, it is important to view the particular epoch in its relationship both to preceding events and to subsequent life. Without entering into detail it is apparent that adverse influences may operate:-

(a) hereditarily.

(b) during embryonic life-e.g. congenital malformations.

(c) during foetal life-e.g. syphilis.

(d) by premature expulsion of the foetus in an immature state.

(e) during birth-e.g. birth-trauma; and failure of the physiological readjustments consequent upon birth.

(f) after birth, during the neonatal period-e.g. infections; lack of adequate nursing skill and care and mismanagement and failure of breast feeding. 